\title{
COSMOLOGICAL MAGNETOHYDRODYNAMIC SIMULATIONS OF CLUSTER FORMATION WITH ANISOTROPIC THERMAL CONDUCTION
}

\author{
M. RuszKowski ${ }^{1,2}$, D. LeE ${ }^{3}$, M. BrüGgen ${ }^{4}$, I. PARRISH ${ }^{5}$, AND S. Peng OH ${ }^{6}$ \\ ${ }^{1}$ Department of Astronomy, University of Michigan, 500 Church Street, Ann Arbor, MI 48109, USA; mateuszr@ umich.edu \\ ${ }^{2}$ The Michigan Center for Theoretical Physics, 3444 Randall Lab, 450 Church Street, Ann Arbor, MI 48109, USA \\ ${ }^{3}$ Department of Astronomy, ASC/Flash Center, University of Chicago, 5640 South Ellis Avenue, Chicago, \\ IL 60637, USA; dongwook@ flash.uchicago.edu \\ ${ }^{4}$ School of Engineering and Science, Jacobs University Bremen, Campus Ring 1, 28759 Bremen 05233, Germany; m.brueggen@ jacobs-university.de \\ ${ }_{5}$ Astronomy Department and Theoretical Astrophysics Center, 601 Campbell Hall, University of California, Berkeley, \\ CA 94720, USA; iparrish@astro.berkeley.edu \\ ${ }^{6}$ Department of Physics, University of California, Santa Barbara, CA 93106, USA; peng@ physics.ucsb.edu \\ Received 2010 October 11; accepted 2011 July 18; published 2011 October 3
}

\begin{abstract}
The intracluster medium (ICM) has been suggested to be buoyantly unstable in the presence of magnetic field and anisotropic thermal conduction. We perform first cosmological simulations of galaxy cluster formation that simultaneously include magnetic fields, radiative cooling, and anisotropic thermal conduction. In isolated and idealized cluster models, the magnetothermal instability (MTI) tends to reorient the magnetic fields radially whenever the temperature gradient points in the direction opposite to gravitational acceleration. Using cosmological simulations of cluster formation we detect radial bias in the velocity and magnetic fields. Such radial bias is consistent with either the inhomogeneous radial gas flows due to substructures or residual MTI-driven field rearrangements that are expected even in the presence of turbulence. Although disentangling the two scenarios is challenging, we do not detect excess bias in the runs that include anisotropic thermal conduction. The anisotropy effect is potentially detectable via radio polarization measurements with LOFAR and the Square Kilometer Array and future X-ray spectroscopic studies with the International X-ray Observatory. We demonstrate that radiative cooling boosts the amplification of the magnetic field by about two orders of magnitude beyond what is expected in the non-radiative cases. This effect is caused by the compression of the gas and frozen-in magnetic field as it accumulates in the cluster center. At $z=0$ the field is amplified by a factor of about $10^{6}$ compared to the uniform magnetic field that evolved due to the universal expansion alone. Interestingly, the runs that include both radiative cooling and thermal conduction exhibit stronger magnetic field amplification than purely radiative runs. In these cases, buoyant restoring forces depend on the temperature gradients rather than the steeper entropy gradients. Thus, the ICM is more easily mixed and the winding up of the frozen-in magnetic field is more efficient, resulting in stronger magnetic field amplification. We also demonstrate that thermal conduction partially reduces the gas accretion driven by overcooling despite the fact that the effective conductivity is suppressed below the Spitzer-Braginskii value.
\end{abstract}

Key words: conduction - galaxies: clusters: general - galaxies: active - instabilities - X-rays: galaxies: clusters

Online-only material: color figures

\section{INTRODUCTION}

Thermal conduction may play an important role in the evolution of the intracluster medium (ICM; e.g., Voigt \& Fabian 2004). Sufficiently strong conduction can offset radiative cooling losses in massive clusters and reduce the energy requirements on active galactic nuclei (AGNs) feedback that is required to prevent overcooling in less massive clusters and groups (see McNamara \& Nulsen 2007 and Norman 2010 for reviews). It has also been suggested that not only can thermal conduction serve as a mechanism for cool core heating but that it is very important for the stability of these systems (Ruszkowski \& Begelman 2002; Guo et al. 2008; Ruszkowski \& Oh 2011). It may also be responsible for setting a critical central entropy threshold below which star formation is possible in cluster cool cores (Voit et al. 2008). The recently discovered bimodality in the distribution of the cluster central entropy (Cavagnolo et al. 2009; Sanderson et al. 2009) may be due to the combination of AGN feedback from the brightest cluster galaxies that stabilizes low entropy clusters and a combination of mergers and thermal conduction that stabilize higher central entropy clusters (Guo et al. 2008; Ruszkowski \& Oh 2010; Parrish et al. 2010). As such, thermal conduction may be important for understanding of the feeding of the most massive black holes in the universe. In cluster outskirts, thermal conduction may flatten the temperature distributions (Parrish et al. 2008), which may have consequences for the cluster mass estimates. This may have a possible impact on precision cosmology as it relies on accurate mass measurements in the most massive clusters.

As a plasma transport process, thermal conduction is closely linked to gas viscosity. Both types of transport processes may explain various $\mathrm{X}$-ray observations. For example, recent Chandra observations of M87 (Werner et al. 2010) show that the temperature in the shells centered on the cluster center is remarkably isothermal. They suggest that such a high degree of isothermality is consistent with effective heat conduction in the tangential direction. Moreover, they also attribute the presence of small-scale metallicity gradients to relatively weak levels of small-scale turbulence, which could be consistent with viscous damping of gas motions. AGNs are known to generate intermittent outflows, causing weak shocks and sound waves (e.g., Fabian et al. 2003; Forman et al. 2005; Finoguenov et al. 2008). The dissipation of the energy contained in these waves may be sufficient to offset radiative cooling of the gas. It has 
been shown observationally (in Perseus: Fabian et al. 2003; in Virgo: Forman et al. 2005) and using numerical simulations (Ruszkowski et al. 2004a, 2004b; Brüggen et al. 2005a) that Spitzer-Braginskii viscosity and/or conduction is sufficient to dissipate such waves and heat the ICM efficiently. Moreover, the compact morphology of buoyantly rising AGNs is consistent with the presence of effective viscosity (Reynolds et al. 2005) although it could also be explained by the magnetic draping effect (Robinson et al. 2004; Ruszkowski et al. 2007, 2008; Dursi \& Pfrommer 2008; O'Neill et al. 2009). Otherwise, the bubbles would be disrupted by Rayleigh-Taylor instabilities. Gas viscosity has also been shown to play a role in shaping the properties of cold fronts caused by the sloshing motion (e.g., ZuHone et al. 2010).

The simple picture of viscosity and conduction is complicated by the presence of magnetic fields that are known to be present in the ICM (Enßlin \& Vogt 2003; Vogt \& Enßlin 2003; Feretti \& Johnston-Hollitt 2004). Magnetic fields suppress thermal conduction in the direction perpendicular to the $B$-field. However, even in the case of highly tangled magnetic fields, the effective thermal conduction can be a substantial fraction the Spitzer conductivity (Narayan \& Medvedev 2001). The anisotropy due to magnetic fields leads to new phenomena with potentially important consequences for the ICM. It has been demonstrated both analytically (Balbus 2000) and numerically (Parrish \& Stone 2005) that the ICM is unstable in the presence of a weak magnetic field and anisotropic thermal conduction when the temperature is increasing in the direction of gravity (magnetothermal instability, MTI). When magnetic fields are partially aligned with the temperature gradient, the heat flow from hotter to cooler regions along the field lines makes such regions more buoyant. This causes the magnetic field to become more aligned with the temperature gradient and leads to the instability and preferentially radial magnetic fields. A similar instability occurs when anisotropic transport of energy via cosmic rays takes place (Chandran \& Dennis 2006; Rasera \& Chandran 2008). More recent analysis shows that the gas is also unstable when the temperature decreases in the direction of gravity in the presence of a background heat flux (HBI instability; Quataert 2008) which has also been verified by numerical simulations (Parrish \& Quataert 2008). The saturated state of the HBI corresponds to the magnetic fields oriented in the direction perpendicular to gravity. Such a field configuration implies effectively vanishing thermal conduction from the hotter outer cluster layers to their cool cores. This accelerates the effective cooling rate in the core. However, the exact topology of the magnetic fields depends also on whether externally imposed turbulence driving is present. This has been recently investigated by Ruszkowski \& Oh (2010) and Parrish et al. (2010), who showed that there exists a critical level of turbulence above which the field can be randomized and the conductive heating to the core restored. Although this analysis was performed for the HBI, similar arguments apply to the MTI. Cluster turbulence may come from AGN outbursts, galaxy motions, and structure formation (major and minor mergers). Dubois et al. (2009) and $\mathrm{Xu}$ et al. (2009) performed a numerical simulation of a magnetized galaxy cluster with AGN feedback. These simulations did not include the effects of non-ideal MHD.

The exact level of turbulence in the ICM is not known but is expected to vary throughout the evolution of a cluster. However, indirect measurements (e.g., Churazov et al. 2003) and upper limits (Sanders et al. 2010) are available. For example, some observational estimates put the ICM velocity at a level of local sound speed (Markevitch et al. 2002; Mahdavi et al. 2007), while others suggest relatively "calm" ICM (Werner et al. 2010). These levels of turbulence are expected theoretically (e.g., Evrard 1990; Nagai et al. 2003; Vazza et al. 2009). Future measurements with the International X-ray Observatory $(I X O)$ will help to determine the level of turbulence in clusters more precisely (Heinz et al. 2011). Therefore, ideally, we need to resort to ab initio cosmological simulations to include the effects of structure formation. Using this approach, we can not only compute the level of the effective thermal conduction in the presence of magnetic fields but we can also simulate the growth of magnetic field. Both MTI and HBI are expected to amplify the fields due to kinematic dynamo action although the efficiency of this process is modest. However, trapping of gravity modes may lead to vorticity growth (Lufkin et al. 1995) and further field amplification (Ruszkowski \& Oh 2011).

Here, we present first cosmological simulations of cluster formation that simultaneously include radiative cooling, magnetic field, and anisotropic thermal conduction. These simulations are a natural extension of our previous work on the role of radiative cooling, magnetic fields, conduction, and viscosity with the FLASH and ATHENA codes that focused on isolated cool cores (Ruszkowski et al. 2004a, 2004b; Brüggen et al. 2005a; Bogdanović et al. 2009; Ruszkowski \& Oh 2010, 2011; Parrish et al. 2010). This work also builds on previous theoretical and numerical efforts to simulate the growth of magnetic field in cluster formation simulations by our group (Brüggen et al. 2005b) with the FLASH code, and other teams (Dolag et al. 1999, 2002) with GADGET; Enzo (Collins et al. 2009), CosmoMHD (Li et al. 2008), RAMSES (Dubois \& Teyssier 2008) as well as the work by Sijacki \& Springel (2006), who considered non-MHD simulations but included viscosity, and Jubelgas et al. (2004) and Dolag et al. (2004) who considered conduction in hydrodynamical SPH simulations. We also note that the field amplification may result from purely kinetic plasma processes (e.g., Schekochihin \& Cowley 2007; Schekochihin et al. 2010; Kunz et al. 2011) and that the largescale turbulence may serve as the energy input for these mechanisms. However, these processes are beyond the scope of this investigation and are not considered here.

The paper is organized as follows. In the next section, we discuss the methods and the code used for the simulations. In Section 3, we discuss our results. Section 4 presents the conclusions. Appendix A discusses the comparison of the anisotropic conduction module with the linear theory predictions. In Appendix B, we briefly discuss the tests of the implementation of the cosmological terms in the MHD equations.

\section{METHODS}

We solve the following set of MHD equations augmented by the cosmological expansion terms

$$
\frac{\partial \rho}{\partial t}+\nabla \cdot(\rho \mathbf{v})=0
$$

$$
\frac{\partial(\rho \mathbf{v})}{\partial t}+\nabla \cdot(\rho \mathbf{v} \mathbf{v}-\mathbf{B B})+\nabla p=-\rho \nabla \phi-2 \frac{\dot{a}}{a} \rho \mathbf{v}
$$




$$
\begin{gathered}
\frac{\partial E}{\partial t}+\nabla \cdot(\mathbf{v}(E+p)-\mathbf{B}(\mathbf{v} \cdot \mathbf{B}))=-\rho \nabla \phi \cdot \mathbf{v} \\
+\frac{\dot{a}}{a}\left[(3 \gamma-1) \rho \epsilon+2 \rho v^{2}\right]+a \mathcal{C}\left(T_{\mathrm{ph}}\right)-\nabla \cdot \mathbf{F}_{\mathrm{ph}} \\
\frac{\partial \mathbf{B}}{\partial t}+\nabla \cdot(\mathbf{v B}-\mathbf{B v})=0
\end{gathered}
$$

where

$$
\begin{gathered}
p=p_{\text {th }}+\frac{B^{2}}{2} \\
E=\frac{\rho v^{2}}{2}+\epsilon+\frac{B^{2}}{2} \\
\Delta \phi=4 \pi G a^{-3}(\rho-\langle\rho\rangle),
\end{gathered}
$$

where $\langle\rho\rangle$ is the comoving mean density and where $p_{\text {th }}$ is the gas pressure and $\epsilon$ is the gas internal energy per unit volume. We assume the adiabatic index $\gamma=5 / 3$ and the mean molecular weight $\mu=0.5$ in the equation of state. In Equation (3), $\mathcal{C}$ represents the cooling rate per unit volume. We use standard tabulated and publicly available cooling curves (Sutherland \& Dopita 1993) for metallicity $Z=0.3 Z_{\odot}$ (Tozzi \& Norman 2001; Böhringer \& Werner 2010). Our simulations do not include star formation or AGN feedback and we set a floor in the cooling function at $0.01 \mathrm{keV}$ in physical units.

The anisotropic thermal conduction heat flux $\mathbf{F}_{\mathrm{ph}}$ is given by

$$
\mathbf{F}_{\mathrm{ph}}=-a^{-1} \kappa\left(T_{\mathrm{ph}}\right) \hat{\mathbf{e}}_{B}\left(\hat{\mathbf{e}}_{B} \cdot \nabla T_{\mathrm{ph}}\right),
$$

where $\hat{\mathbf{e}}_{B}$ is a unit vector pointing in the direction of the magnetic field and $\kappa$ is the Spitzer-Braginskii conduction coefficient given by $\kappa(T)=4.6 \times 10^{-7} T^{5 / 2} \mathrm{erg} \mathrm{s}^{-1} \mathrm{~cm}^{-1} \mathrm{~K}^{-1}$. In the above equation and in all equations below all variables with the "ph" subscript denote physical quantities. Following Cowie \& McKee (1977) we included the effect of conduction saturation whenever the characteristic lengthscale associated with the temperature gradient exceeds the mean free path, though in the bulk of the ICM this effect is not significant. We also imposed an upper limit on conduction such that $K \equiv a^{6} \kappa /\left(c_{v} \rho\right)$, where $c_{v}^{-1}=(\gamma-1) \mu \mathrm{m}_{\text {prot }} / k_{\text {boltz }}$ (the extra factor of $a$ comes from the prefactor in Equation (8)). We set $K<K_{\max }=5 \times 10^{32} \mathrm{~cm}^{2} \mathrm{~s}^{-1}$. This ceiling was introduced in order to prevent extremely small diffusive timesteps. We found that changing $K_{\max }$ to larger values did not affect our results significantly. Only a relatively limited volume far from the cluster center was subject to this upper limit.

We also note that the effective conduction may be limited in the outer regions of the cluster for physical reasons (Medvedev 2007). Moreover, around or beyond the virial radius, the electron-proton energy equilibration timescales start to exceed the dynamical or buoyancy timescale. In this region, the MHD approximation starts to break down and the MTI growth rates are reduced. However, at smaller radii, the equilibration timescale due to Coulomb collisions is comparable to or shorter than the buoyancy timescale, some thermal coupling between electrons and protons begins to be possible, and the MHD analysis applies at least approximately (Dougal \& Goldstein 1958; Padmanabhan 2000). Finally, there are independent observational arguments for fast equilibration in the ICM. Specifically, Markevitch \& Vikhlinin (2007) detected a sharp electron temperature jump in the post-shock region in the Bullet Cluster and used this observation to suggest that the electron-proton equilibration is much shorter than the collisional timescale. The inferred equilibration timescale in the post-shock ICM is at least five times shorter than the Coulomb equilibration time and may even be consistent with being instantaneous. While the issue of equilibration is clearly an open research topic, here we work under the simplifying assumption that equilibration is instantaneous and that the MHD approximation is appropriate, and then investigate the consequences of these assumptions.

The most stringent limitation on the timestep $d t$ in the simulation was due to the diffusive process with $d t \sim(\Delta x)^{2} / K$. This timestep was global, i.e., it was set by the most stringent constraint across all blocks and then used to evolve the entire computational domain. The maximum resolution that we could afford computationally was seven levels of refinement for blocks consisting of 16 zones on a side. That is, the effective resolution was $\sim 31 h^{-1} \mathrm{kpc}$. The strong limit imposed on the timestep can be avoided by employing implicit integration methods. We are now implementing such methods in the FLASH code (D. Lee et al. 2012, in preparation). This approach will significantly speed up the computations and will allow us to perform simulations for a range of cluster masses (M. Ruszkowski et al. 2012, in preparation).

Equations (1) through (8) were obtained by starting from the MHD equations and applying cosmological expansion transformation for the spatial gradients and the following variable transformations:

$$
\begin{gathered}
\rho=a^{3} \rho_{\mathrm{ph}} \\
p=a p_{\mathrm{ph}} \\
T=a^{-2} T_{\mathrm{ph}} \\
\rho \epsilon=a \rho_{\mathrm{ph}} \epsilon_{\mathrm{ph}} \\
\mathbf{B}=a^{1 / 2} \mathbf{B}_{\mathrm{ph}},
\end{gathered}
$$

where $a$ is the cosmological expansion factor. The velocity vector $\mathbf{v}=\dot{\mathbf{x}}$, where $\mathbf{x}=a^{-1} \mathbf{r}$ is the code position vector and $\mathbf{r}$ is the physical position vector. These transformations together with Equations (1)-(7) lead to correct scalings of all quantities with the expansion factor. For example, $T_{\mathrm{ph}} \propto a^{-2}$ and $B_{\mathrm{ph}} \propto a^{-2}$, which ensures the conservation of the magnetic flux. Note that the variable transformation adopted here is somewhat different than, e.g., that in Li et al. (2008) in the CosmoMHD code, but the scalings of the physical quantities with redshift are correct in both cases. The cosmological terms were included using a method analogous to that employed by Ricker et al. (2000). Specifically, we computed the updates to all variables due to the cosmological expansion terms by finding the exact solutions to a modified set of Equations (1)-(4) that retained only the time derivative terms. Tests of the implementation of the cosmological terms are presented in Appendix A.

We used initial conditions very similar to the publicly available initial conditions for the Santa Barbara cluster (Frenk et al. 1999). Santa Barbara conditions correspond to initial $3 \sigma$ density perturbations spread over $10 \mathrm{Mpc}$, Hubble constant of $50 \mathrm{~km} \mathrm{~s}^{-1} \mathrm{Mpc}^{-1}$, and flat geometry in a matter-dominated 
universe $(\Lambda=0)$ in a 64 Mpc computational box, and result in the formation of a cluster characterized by $r_{\mathrm{vir}} \sim 2.7 \mathrm{Mpc}$. Our initial conditions have normalizations of the initial background fluctuations different from those employed in the original Santa Barbara project, which results in a slightly smaller virial mass. However, we also performed an adiabatic test simulation with identical initial conditions and obtained fully consistent results. We employed periodic boundary conditions. We note that the results are not sensitive to the initial temperature and strength of the magnetic fields as long as they are small, which is the case in our simulations. In particular, the final strength of the magnetic field may be simply rescaled depending on the initial field, as long as the initial field is weak. The initial magnetic field was assumed to be constant in space. While in isolated cluster simulations the initial topology of the magnetic field can affect the instability growth rate, the frozen-in magnetic field in the cosmological simulations is randomized by structure formation motions well before the hot virialized and relaxed structures are formed. Since early on thermal conduction is not expected to be very important, the initial field topology is not expected to play a crucial role. In fact, it has been demonstrated using cosmological MHD simulations without transport processes that the results were indeed not sensitive to the exact topology of the magnetic field in the statistical sense (Dolag et al. 2002; Brüggen et al. 2005b). Therefore, for simplicity we assume an initial magnetic field that is pointing in the same direction and is uniform. We set the initial strength of the magnetic field to $10^{-11} \mathrm{G}$, i.e., the initial physical magnetic field strength was $10^{-11}(1+z)^{1 / 2} \mathrm{G}$ at the initial redshift of $z=20$.

The simulations were performed with the adaptive mesh refinement (AMR) FLASH code. FLASH is a publicly available code that was developed in part by the DOE-supported ASC/Alliance Center for Astrophysical Thermonuclear Flashes at the University of Chicago. It is a modular, parallel simulation code capable of handling general compressible flow problems found in many astrophysical environments. The code is parallelized using the Message-Passing Interface (MPI) library and the HDF5 or PnetCDF library for parallel I/O to achieve portability and scalability on a variety of different parallel computers.

The new directionally unsplit staggered mesh MHD solver (Lee \& Deane 2009; Lee et al. 2009) is based on a finite-volume, high-order Godunov method combined with a constrained transport scheme which ensures divergence-free magnetic fields. Tests of the module demonstrate that it is very robust and significantly outperforms the previously implemented MHD eight-wave solver in FLASH.

We implemented the anisotropic conduction unit following the approach of Sharma \& Hammett (2007). More specifically, we applied a monotonized central limiter to the conductive fluxes. This method ensures that anisotropic conduction does not lead to negative temperatures in the presence of steep temperature gradients. We verified that the module predicts correct MTI growth rates. Details of these tests are discussed in Appendix B.

\section{RESULTS}

\subsection{Temperature Distribution}

Figure 1 shows cross sections through the temperature distribution in the cluster. All panels correspond to $32 h^{-1} \mathrm{Mpc}$ on a side. The minimum and maximum values of the temperature are the same in all panels. The top row is for the non-conductive cases while the bottom row is for the runs with anisotropic thermal conduction. Left columns are for non-radiative simulations and the right one for the runs with radiative cooling. The upper right panel clearly shows a well-developed cool core. Comparison of the top and bottom rows shows that conduction is efficient in smearing out the fine structure details in the temperature distribution. The cool core in the conductive case is partially heated by thermal conduction from the hotter outer layers of the cluster but it does not become isothermal.

In Figure 2, we show the temperature profiles of the cluster for all four cases shown in Figure 1. The profiles are not densityweighted. The dashed green curve is for the non-radiative run, solid dark blue for anisotropic conduction, dashed light blue for radiative cooling, and solid red is for radiative cooling with anisotropic conduction. The solid black line is for the case with radiative cooling and isotropic conduction at the level of $1 / 3$ of the Spitzer value. The adopted bin size was $\sim 31 h^{-1} \mathrm{kpc}$. For larger bin sizes, the comparison between the temperature profiles and the temperature maps shown in Figure 1 is more difficult; unless stated otherwise, other one-dimensional profiles presented below use a bin size approximately three times larger, which is especially important for the observables inferred from the vector quantities as they tend to exhibit larger fluctuations. The effect of thermal conduction on the temperature profiles is mild but noticeable. This is partially due to the fact that magnetic fields reduce the effective level of conduction below the Spitzer value. The effect is more pronounced in Jubelgas et al. (2004) who use full isotropic Spitzer conductivity in cosmological simulations. Radiative cooling has much stronger effect on the temperature distribution. The run with cooling and $1 / 3$ of isotropic Spitzer conduction shows a slightly higher central temperature than its anisotropic conduction counterpart. This is consistent with the fact that, on average, the fields are slightly more tangential in the center in the latter case and tend to suppress conduction below $1 / 3$ the Spitzer value. The geometry of the field is discussed in more detail in Section 3.2 below. Interestingly, the cooling runs show excess temperature at larger radii. We interpret this effect as a consequence of the combination of the increased role of shocks in the cooling ICM that generate entropy and "adiabatic" compression (e.g., Lufkin et al. 2000). Both of these effects are more important in the presence of radiative losses in the cluster center. The rapid cooling "pulls" the outer cluster layers toward the center and heats up the ICM. In the presence of cooling, the shocks are stronger and the heating of the gas more efficient. This effect is illustrated in Figure 3 where we show the entropy profiles. The meaning of the curves is the same as in Figure 2. Figure 3 shows that the radiative runs exhibit elevated entropy at $r \gtrsim 300 h^{-1} \mathrm{kpc}$.

We point out that the temperature profile declines with the distance from the cluster center even in the runs that include conduction. This is possible when the virialization shocks heat the gas faster than conduction can remove heat.

\subsection{Statistical Properties of Magnetic and Velocity Field Orientations}

In the very central parts of the cluster the suppression of the effective conduction is further enhanced by a partially tangential ordering of the fields. This effect is shown in the left panel in Figure 4, where we plot the absolute value of the radial component of the magnetic field vector (left panel) and anisotropy parameter $\beta$ for the magnetic field (middle panel). This quantity is defined as $\beta=1-\sigma_{b t}^{2} / 2 \sigma_{b r}^{2}$, where $\sigma_{b t}$ and 


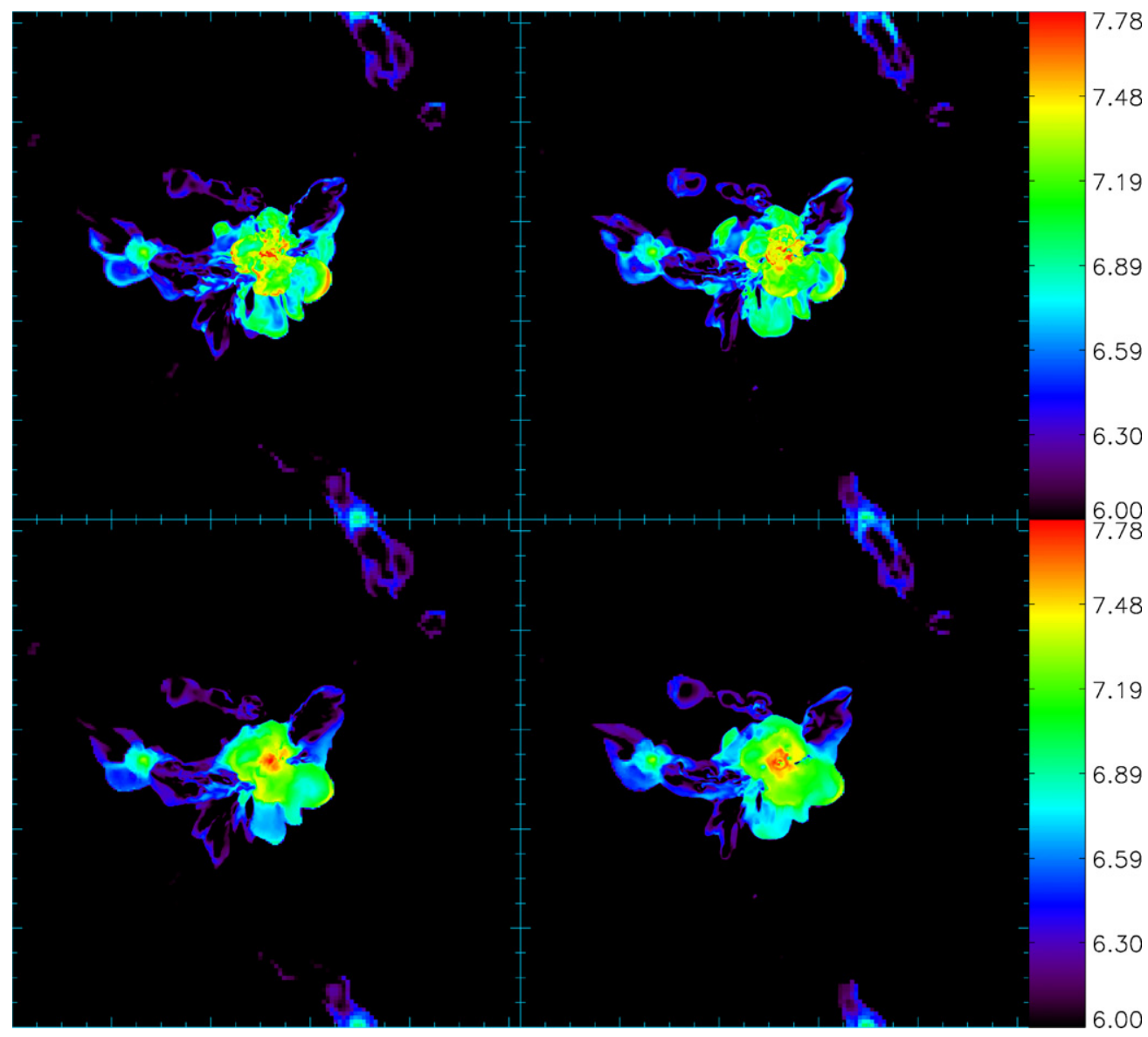

Figure 1. Cross sections through the temperature distribution in the cluster at $z=0$. All panels correspond to $32 h^{-1} \mathrm{Mpc}$ on a side. The top row is for the non-conductive cases while the bottom row is for the runs with anisotropic thermal conduction. The left column corresponds to non-radiative simulations and the right one is for the runs with radiative cooling. The minimum and maximum values of the temperature are the same in all panels. Color bars show the logarithm of the temperature in Kelvins. This figure illustrates the relative differences between simulations including different physics processes. Their effect is quantified in Figure 2 , which shows temperature profiles.

(A color version of this figure is available in the online journal.)

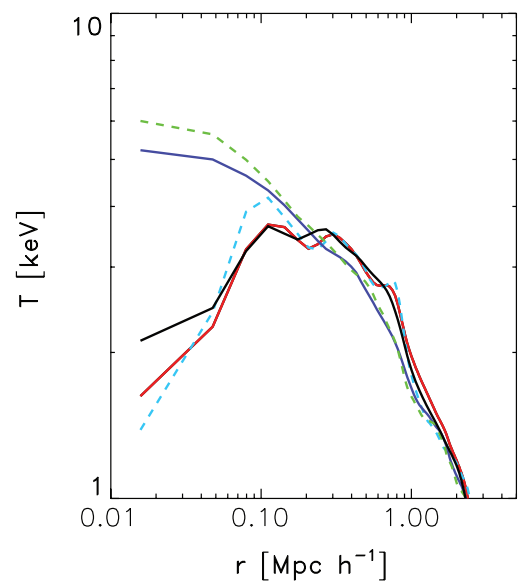

Figure 2. Temperature profiles of the cluster. The dashed green curve is for the adiabatic run, solid dark blue for anisotropic conduction, dashed light blue for radiative cooling, and solid red is for radiative cooling with anisotropic conduction. The solid black line is for the case with radiative cooling and isotropic conduction at the level of $1 / 3$ of the Spitzer value. The profiles are not density-weighted.

(A color version of this figure is available in the online journal.)

$\sigma_{b r}$ are the transverse and radial magnetic field dispersions, respectively. For example, $\sigma_{b t}=\left\langle B_{t}^{2}\right\rangle^{1 / 2}$ and the definition of $\sigma_{b r}$ is analogous. The meaning of the curves is the same as

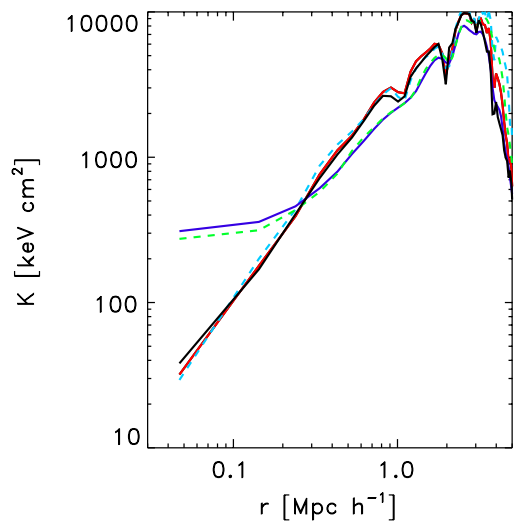

Figure 3. Entropy profiles of the cluster. The meaning of the curves is the same as in Figure 2. Entropy is defined as $K=k_{\text {boltz }} T / n_{e}^{2 / 3}$ and the plot units are

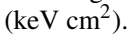

(A color version of this figure is available in the online journal.)

in Figure 2. For the absolute value of the radial component of the magnetic field, the isotropic case corresponds to 0.5. For magnetic anisotropy, the isotropic case corresponds to vanishing $\beta$, and the tangential and radial cases to negative and positive $\beta$, respectively. Horizontal dotted lines correspond to isotropic configurations. At large radii there appears to be a slight radial 

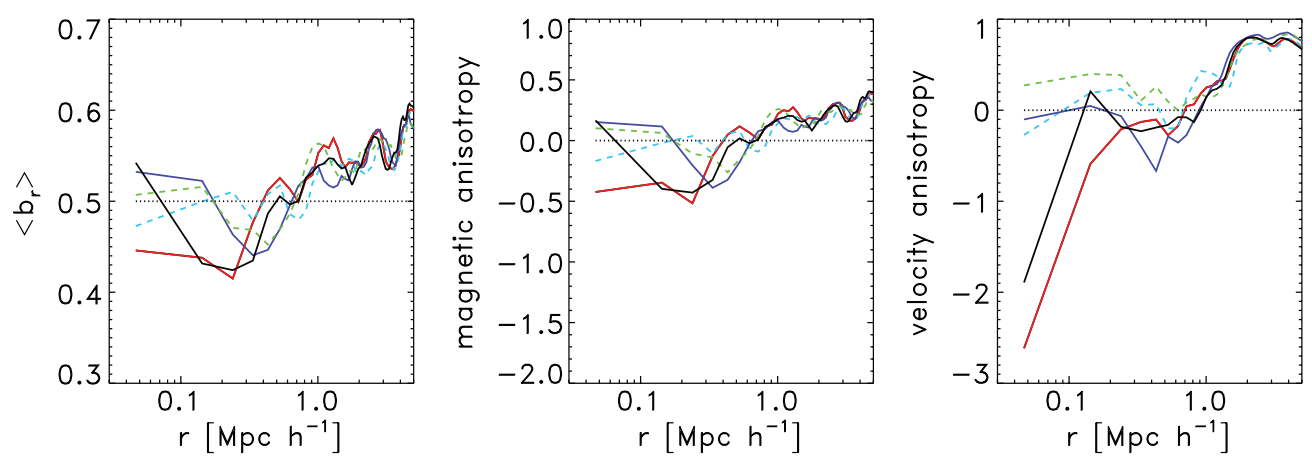

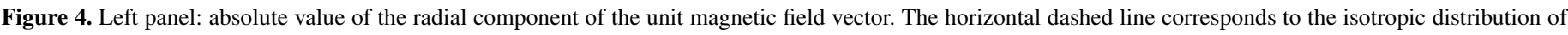

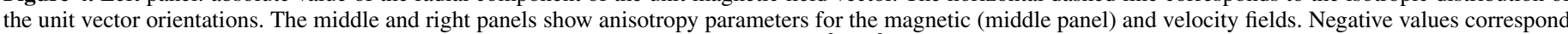

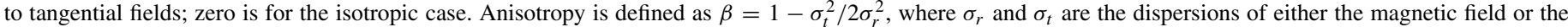
velocity field. The color coding of all curves is the same as in Figure 2. Horizontal dotted lines correspond to isotropic configurations.

(A color version of this figure is available in the online journal.)

bias in the orientation of the magnetic field. This could be attributed to the accretion along the filaments as the magnetic fields are expected to be locally preferentially tangential to these structures (Brüggen et al. 2005b) or to inhomogeneous radial flows in general. The right panel of Figure 4 shows the anisotropy parameter $\beta_{v}$ for the velocity field. The definition of this quantity is analogous to that for the magnetic fields with velocity dispersions replacing the magnetic field dispersions. However, the velocity dispersion is measured with respect to the mean streaming velocity of the cluster. This figure bears an interesting resemblance to its magnetic counterpart. There is an even stronger tangential bias in the velocity field in the radiative run with anisotropic thermal conduction than in the magnetic anisotropy parameter for the same run. However, there is a significant scatter in these quantities and firm conclusion about the trend could be drawn by averaging over many clusters. The exact values also depend on such factors such as the definitions of the cluster center and its bulk velocity, and whether the quantities are mass-weighted or not. Nevertheless, some similarities in these quantities are not unexpected, due simply to the fact that the magnetic field is frozen to the gas and follows it. However, unlike the velocity field, the magnetic field has a "memory" of past gas displacement, so the two anisotropy parameters are not expected to be identical. Intermediate radii tend to have relatively more tangential velocity field.

\subsubsection{Radial Bias in the Velocity and Magnetic Fields}

As argued in Sharma et al. (2009) and Ruszkowski \& Oh (2010), the ability of the MTI to reconfigure the magnetic field depends on the level of externally-driven turbulence. In the current case, the turbulence is driven by the structure formation motions. Strong turbulence can significantly affect the MTI but some residual radial bias may be present in the field. McCourt et al. (2011) showed that even for strong turbulence driving, the MTI operates and significantly contributes to the velocity power spectrum on scales larger than the outer turbulence driving scale $L_{\text {out }}$. On scales smaller than $L_{\text {out }}$, the power spectra in the conduction and adiabatic runs are nearly identical. As these isolated box experiments considered a relatively small outer driving scale ( $L_{\text {out }}=h_{\text {pres }} / 16$, where $h_{\text {pres }}$ is the pressure scale height in a box $h_{\text {pres }} / 2$ high), larger outer driving scales may reduce the parameter space where the MTI operates.

If the Froude parameter is less than unity, then the turbulence does not reset the magnetic field configuration established by the MTI. We define the Froude number here as $F r=$
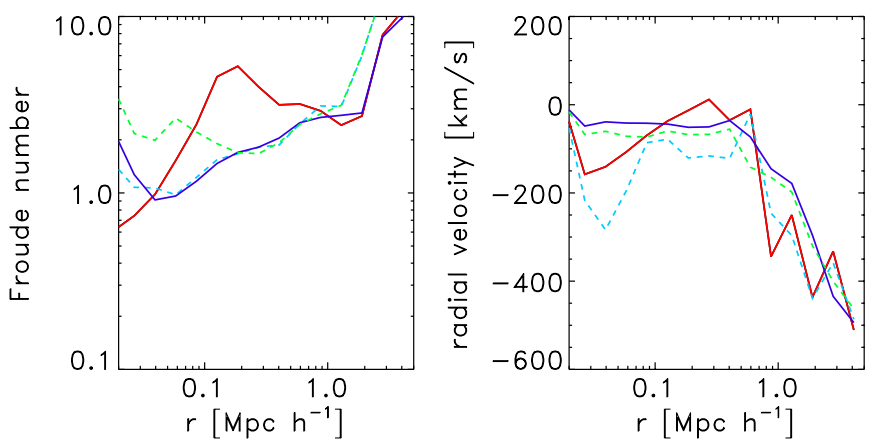

Figure 5. Froude number $F r$ as a function of the distance from the cluster center (left panel) and the radial component of the gas velocity. The radial velocity plot shows net mass-weighted infall velocity. Both panels are plotted using 15 spherical shells of logarithmic width 0.168 in the range $15 h^{-1} \mathrm{kpc} \leqslant r \leqslant$ $5 h^{-1}$ Mpc. The color coding of the curves is the same as in Figure 2. For $\mathrm{Fr}>1$ the turbulence is expected to begin to randomize the magnetic field configuration that would otherwise be established by the instabilities.

(A color version of this figure is available in the online journal.)

$\sigma / \omega_{\mathrm{BV}} L$, where $\sigma$ is the gas velocity dispersion and $L$ is the characteristic lengthscale. Here we define it entirely for reference purposes as the pressure scale height $L=P / \delta P$ in all runs. The characteristic frequency $\omega$ of the gas perturbed from its equilibrium state is

$$
\omega^{2}=\frac{g}{r}\left(\frac{3}{5}\left|\frac{d \ln S}{d \ln r}\right|,\left|\frac{d \ln T}{d \ln r}\right|\right),
$$

where $g$ is the total gravity and $S=k_{b} T / n_{e}^{2 / 3}$ is the gas entropy. The first term in brackets on the right side of Equation (14) is for the standard Brunt-Väisälä frequency while the second term is for its MHD equivalent in the presence of anisotropic thermal conduction. The Froude number as a function of the distance from the cluster center is shown in the left panel in Figure 5. The meaning of the curves is the same as in Figure 2. For the runs without anisotropic conduction we used the standard Brunt-Väisälä frequency to evaluate the Froude parameter and for those that include anisotropic thermal conduction we used the MHD equivalent of the Brunt-Väisälä frequency (see Equation (14) above). As can be seen in this figure, the Froude parameter typically exceeds unity. This suggests that the instabilities should be significantly affected by the turbulence. However, the estimate of $\mathrm{Fr}$ is subject to uncertainties because of the arbitrariness of the choice of the characteristic turbulence scale. Therefore, the $\mathrm{Fr}$ parameter should only be taken 
as a rough guide to the impact of the turbulence. We also experimented with other definitions of $L(=100 \mathrm{kpc}$, hydrostatic pressure scale height, and $\sigma /|\nabla \omega|$, where $\sigma$ is the velocity dispersion and $\omega$ is the vorticity). In all cases, typical values of the Fr number tend to exceed unity.

Despite the fact that the Froude number tends to exceed unity, the velocity and magnetic fields indicate clear radial bias for $r \gtrsim 0.8 h^{-1} \mathrm{Mpc}$ in Figure 4. At $r \sim 0.8 h^{-1} \mathrm{Mpc}$ the result is consistent with no bias even though the timescales for the MTI development are short (see the next section). Radial bias in the magnetic field in the part of the ICM where the temperature declines with distance from the center could be caused by the MTI. However, the radial bias is present at large distances from the cluster center where the contribution of turbulence to the pressure support is strongest, which tends to isotropize the fields. Thus, a plausible and simple explanation for the radial bias is that it is due to inhomogeneous radial gas flows either in the bulk of the ICM or through the filaments in more distant parts of the cluster. We verified that indeed some residual net infall velocity is present at larger radii in all four runs, i.e., independently of whether anisotropic thermal conduction is included. This effect is shown in the right panel of Figure 5 where the magnitude of the radial component of the gas velocity systematically increases at large distances from the center. The meaning of all curves is again the same as in Figure 2. This is also consistent with the radial bias in the velocity field that increases with the distance from the cluster center (right panel in Figure 4 discussed above). However, there is no excess magnetic and velocity anisotropy bias in the runs with anisotropic thermal conduction. Nevertheless, we point out that it is difficult to disentangle the effect of the MTI and the inhomogeneous radial flow on the orientation of the magnetic fields. Moreover, a definite statement about the likelihood of the magnetic field reorientation due to the MTI in the bulk of the cluster volume will have to await a systematic study of more than just one cluster as the dynamical states and properties of the ICM turbulence (such as the effective outer driving scale) will be different in other clusters. Here we discuss the result of a pilot study that forms the basis for future investigation in this direction.

The radiative run without conduction (dashed light blue line, right panel in Figure 5) exhibits net accretion even at smaller radii which is due to the cooling flow. It is worth noting that, in the radiative run with anisotropic conduction, the weak net infall in the central cluster regions is still present but it is significantly reduced compared to the cooling-only case (see the solid dark blue line for the radiative run with conduction and the dashed light blue line for the radiative run for $\left.r<0.5 h^{-1} \mathrm{Mpc}\right)$.

\subsubsection{Observations of Magnetic Field Orientation in Clusters}

We also note that Pfrommer \& Dursi (2010) reported on radio polarization measurements in the Virgo cluster based on the observations of magnetic field draping around the cluster galaxies. Pfrommer \& Dursi (2010) suggests that the polarization pattern in the vicinity of the magnetic "tracer" galaxies interacting with the ICM is consistent with predominantly radial fields at large distances from the cluster center. They further pointed out that this observation is consistent with the predictions of the MTI. For this mechanism to work, the MTI would have to either reestablish the radial magnetic field bias on a timescale at least comparable to the dynamical timescale of the orbiting "tracer" galaxies or the properties of the galaxies must be such that they
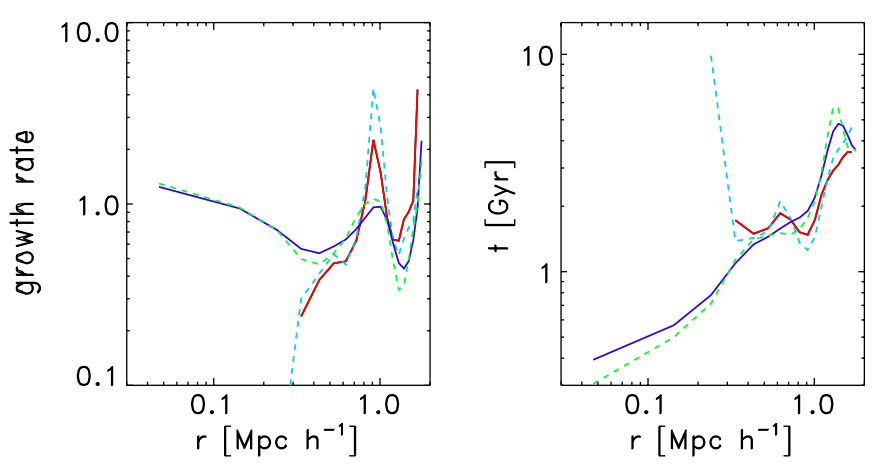

Figure 6. Left: MTI growth rates in units of Brunt-Väisälä frequency. Right: MTI timescales. In both panels the color coding of the curves is the same as in Figure 2. For the runs without anisotropic conduction the rates are inferred from the density and temperature distributions via the post-processing of the simulation data.

(A color version of this figure is available in the online journal.)

do not dramatically stir the ICM in a continuous fashion and/or that a significant time passed since the last major merger. Such instability growth rates may be possible but whether they are realized depends on the details of the dynamical structure of the ICM.

\subsubsection{MTI Growth Rates and Timescales}

In Figure 6 (left panel), we show the MTI growth rates in units of the Brunt-Väisälä frequency. For the runs without anisotropic conduction, the rates shown are those that would have been present had conduction been included. That is, in this case the MTI rates are computed via the post processing of the simulation data. In all cases, the growth rates were obtained from the MTI dispersion relation, taking into account the slope of the temperature and entropy profiles. As by definition we are only interested in those fields that can be reoriented in the radial direction due to the MTI, we assumed tangential fields when computing the MTI growth rates. For radial fields the MTI growth rate would be infinite. Furthermore, we considered perturbation wavelength equal to the radius. Shorter fluctuation wavelengths would result in somewhat faster growth rates. The right panel in Figure 6 shows the corresponding MTI instability growth timescales. Both panels in this figure show that the MTI instability has had time to develop across a wide range of distances from the cluster center in the non-radiative case. Nevertheless, the discussion of the Froude parameter above suggests that, at least in the cluster considered here, the instability might have been overwhelmed by the stirring motions in the ICM. A mild radial bias in the orientation of the magnetic field (and velocity) is present in the simulations at large radii but it is consistent with being due to either gas inflow or preferentially radial substructure motions. However, we reiterate the point made earlier that it is difficult to disentangle the inhomogeneous radial flow from residual MTI. In the runs with radiative cooling, the timescales in the more central parts of the cluster are long (dashed blue line corresponds to high values of the MTI growth timescale for $r \lesssim 0.2 h^{-1} \mathrm{Mpc}$ and the solid red line in the same region is not shown but corresponds to very long timescale). This is caused by the flatness of the temperature distribution, which makes the gas neutrally buoyant. As we explain below, this effect leads to an enhanced magnetic field amplification in this region when anisotropic conduction is included in the simulation. The same stirring motions that tend to suppress the MTI in the non-radiative case are responsible for 


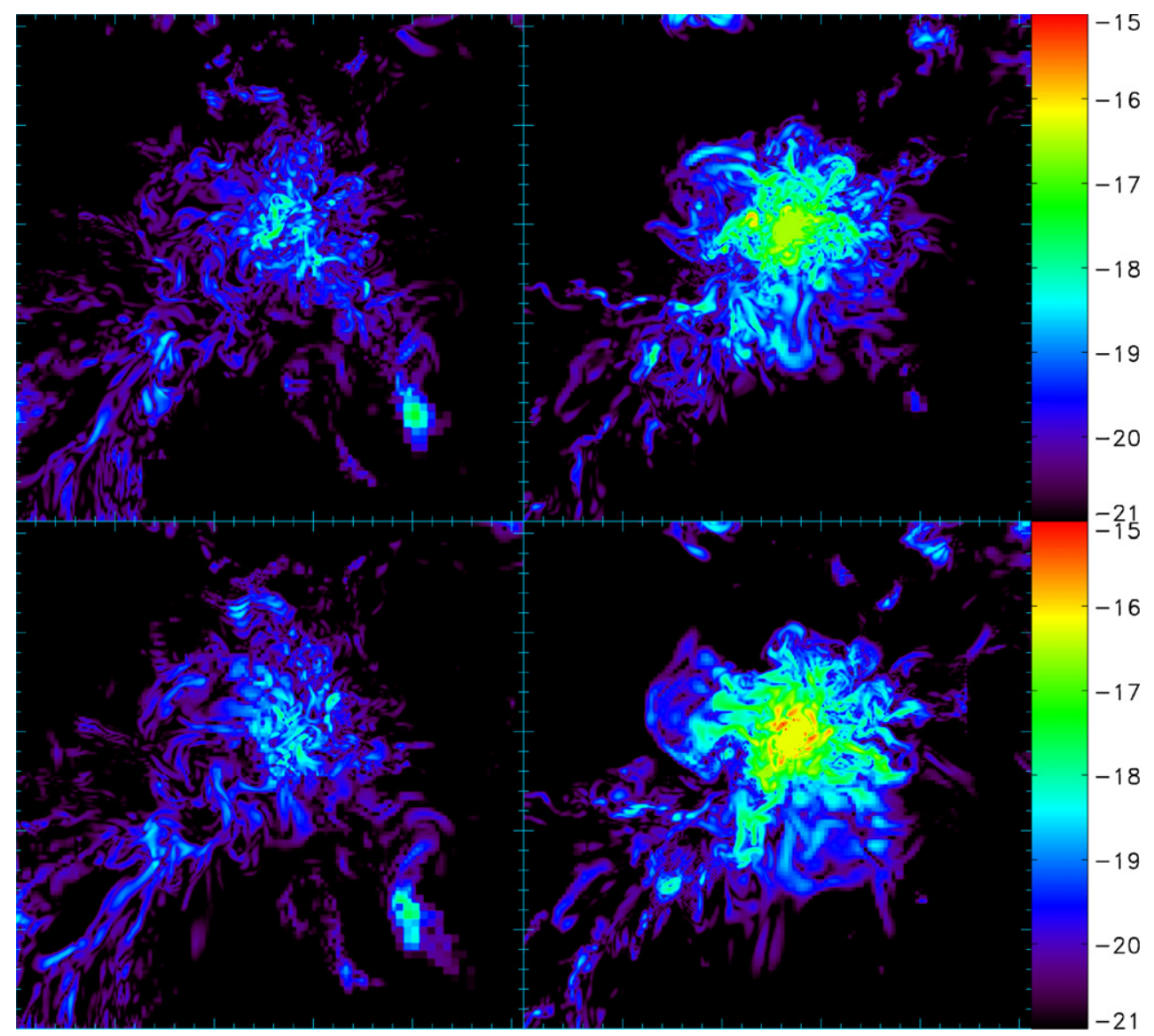

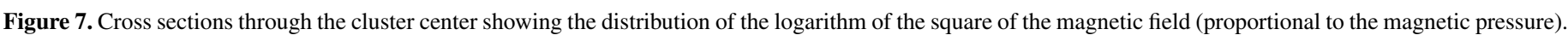

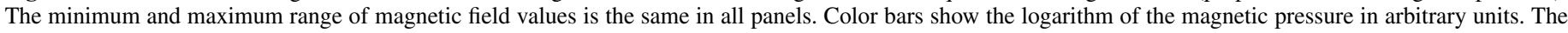

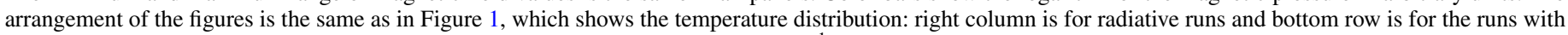
anisotropic thermal conduction. All panels show the central region that measures $8 h^{-1} \mathrm{Mpc}$ on the side.

(A color version of this figure is available in the online journal.)

driving unimpeded stirring in the radiative one, thus amplifying the magnetic fields. However, even in the radiative runs, the MTI growth rates are reasonably short beyond $\sim 0.3 h^{-1} \mathrm{Mpc}$ from the center. We also point out that the slopes of the temperature distribution in the runs with radiative cooling are steeper at higher redshifts, and therefore a wider range of radii was in the unstable regime earlier on.

\subsection{Magnetic Field Amplification}

Cross sections through the cluster center showing the distribution of the logarithm of the square of the magnetic field (proportional to the magnetic field pressure) are shown in Figure 7. The minimum and maximum ranges of magnetic field values are the same in all panels. The magnetic field is in Gauss. However, which we emphasize that, technically speaking, the field values can be arbitrarily rescaled by varying the initial magnetic field as long as the field is weak (which is the case in our simulations). The arrangement of the figures is the same as in Figure 1, which shows the temperature distribution: right column is for radiative runs and bottom row is for the runs with anisotropic thermal conduction. All panels show the central region, which measures $16 \mathrm{Mpc}$ on the side. The magnetic field is clearly amplified in the cluster center in all four cases. The amplification is much stronger in the radiative runs due to the compression of the cool gas and the magnetic field that is frozen into it. This is consistent with the findings of Dubois \& Teyssier (2008) who performed MHD simulations of cluster formation with radiative cooling. Interestingly, the radiative cooling run with anisotropic thermal conduction (bottom right panel) shows even stronger magnetic field amplification than the radiative cooling run. This result is not unexpected. The additional amplification occurs in the region where the temperature gradient is significantly flatter than in other runs. The temperature flattening occurs outside the central cool core and up to $\sim 0.5 h^{-1} \mathrm{Mpc}$ from the cluster center. The nature of convective motions changes in the presence of anisotropic thermal conduction. When the temperature profile flattens, the ICM tends to become neutrally buoyant. This means that the restoring forces in the fluid diminish and the substructure infall becomes the main engine for driving the unimpeded mixing of the gas. Consequently, this results in more efficient winding up and amplification of the magnetic field frozen into the gas via the kinematic dynamo effect.

In order to better quantify the amplification of the field, we also plot the distribution of the magnetic field along the line passing through the cluster center. This is shown in the left panel of Figure 8. The color coding of the curves is the same as in Figure 2 . The runs with cooling boost the field by over two orders of magnitude beyond the amplification seen in the non-radiative cases. The top horizontal line denotes the physical field at the initial redshift $(z=20)$ and the bottom one is for the value of the field $B_{o}$ that would result from cosmological expansion down to $z=0$ without any structure formation effects (the magnetic field in this figure is dimensionless and expressed in units of $B_{o}$ ). These reference levels show that the magnetic field in clusters 

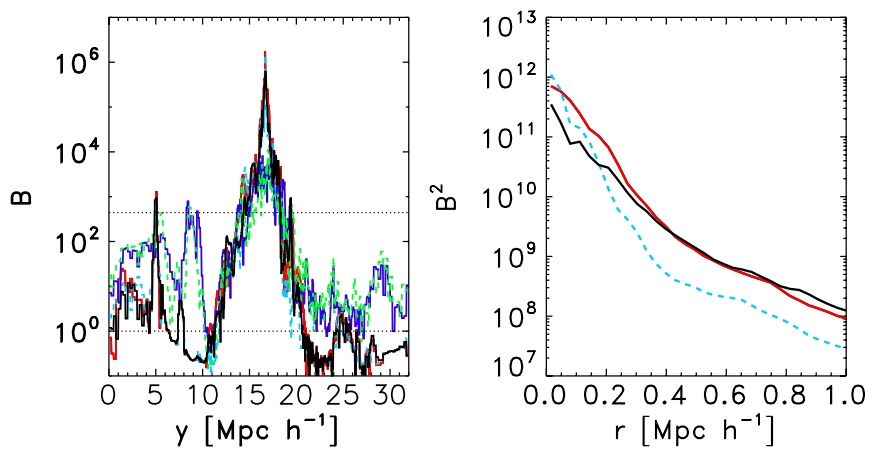

Figure 8. Distribution of the magnetic field along the line passing through the cluster center (left panel). The color coding of the curves is the same as in Figure 2. The top horizontal line denotes the physical field at the initial redshift $(z=20)$ and the bottom one is for the value of the field that would result from cosmological expansion down to $z=0$ without any structure formation effects. The right panel shows the central magnetic pressure profiles. Here, the solid red line is for the anisotropic conduction and cooling while the dashed light blue line is for the run that includes only radiative cooling. Only runs with cooling are shown in this panel.

(A color version of this figure is available in the online journal.)

is boosted beyond the initial physical field by over an order of magnitude. The boost in the strength of the magnetic field in the adiabatic case is $\sim 10^{4}$ compared to the value a uniform magnetic field would have at $z=0$. The magnitude of this amplification is consistent with that obtained by Dolag et al. (1999, 2002), and in our earlier work (Brüggen et al. 2005b) where the numerical resolution was higher. Simple scaling arguments show that this field strength exceeds the field expected from the magnetic flux freezing arguments. Specifically, for flux freezing, we have for the ratio of physical field strengths $B(z=0) / B\left(z=z_{\text {ini }}\right)=\left[\rho(z=0) / \rho_{\text {ini }}\right]^{2 / 3}$. For a typical cluster, the final overdensity is $\sim 10^{3}$ above the current critical density. Thus, for the cosmology relevant to the Santa Barbara run, we have $B(z=0) / B\left(z=z_{\text {ini }}\right)=\left[10^{3} /\left(1+z_{\text {ini }}\right)^{3}\right]^{2 / 3} \sim 0.2$, which is much smaller than the amplification factor of a few $\times 10$ that we see in the adiabatic simulation. This shows that the final magnetic field in the adiabatic simulation is additionally boosted by the shearing motions during the cluster formation. We point out that the final magnetic fields at the cluster center in the cooling run and the run with anisotropic conduction with cooling are boosted even further. The magnetic fields are $\sim 3 \times 10^{3}$ times stronger than the physical field at the initial redshift of $z=20$ (or equivalently, over $10^{6}$ stronger than a uniform field would have at $z=0$ due to the universal expansion alone, or about a hundred times stronger than in the adiabatic run). Such levels of magnetic field amplification are also in qualitative agreement with the higher resolution simulations of Dubois \& Teyssier (2008), who performed adiabatic and radiative runs without thermal conduction. Such field strengths are below the MTI and HBI suppression values. Thus, for the initial field strengths considered here, the suppression of these instabilities by magnetic tension does not play a significant role.

Outside the cluster center, the field at $z=0$ is reduced compared to its initial value. This is simply the result of the cosmological expansion. However, the field far from the cluster center is still somewhat higher than the uniform field evolved down to $z=0$ due to the universal expansion alone. This could be attributed to mild amplification in filaments or smaller gravitationally bound structures. It is interesting to note that the runs with radiative cooling show even weaker fields far from the cluster center. This is expected as the gas tends to cool down
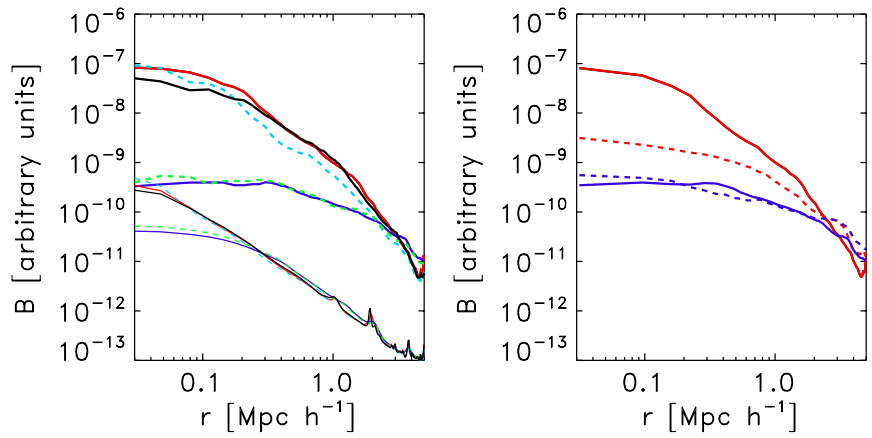

Figure 9. Left panel: magnetic field profiles for the case including anisotropic conduction and radiative cooling (red curves), and non-radiative case with anisotropic conduction. The solid lines are for the seven levels of refinement, and the dashed ones for the six levels of refinement. Right panel: profiles of magnetic field $B$ for the seven levels of refinement. The color and symbol coding of all thick curves are the same as in Figure 2, and thin curves correspond to the thick curve counterparts computed under the assumption that the amplification of the magnetic field $B_{\text {comp }}$ comes entirely from gas compression (i.e., $B_{\text {comp }} \propto \rho^{2 / 3}$ ).

(A color version of this figure is available in the online journal.)

and move closer to the center of the cluster, filaments, and halos, amplifying the field in these regions at the expense of the rest of the volume.

As mentioned above, the field amplification in the cooling run with anisotropic conduction shows stronger field amplification compared to its non-conductive counterpart (Figure 7, compare the top and bottom panels in the right column). In order to better illustrate this effect, we zoom in on the central cluster regions and show central magnetic pressure profiles in the right panel of Figure 8. The solid red line is for the anisotropic conduction and cooling while the dashed light blue line is for the run that includes only radiative cooling. The solid black line is for the cooling case with isotropic conduction at $1 / 3$ Spitzer level. It is clear from this figure that the magnetic field pressure in the runs with conduction and cooling is stronger by up to $\sim 10$ times compared to the cooling-only run. This is because of the combination of two factors: (1) the restoring forces in the conducting fluid are proportional to the temperature gradient rather than the entropy gradient (even if conduction is isotropic; McCourt et al. 2011) and (2) the temperature gradients are shallower than the entropy gradients (cf. Figures 2 and 3). Thus, it is easier to mix the ICM, and amplify the magnetic fields, in the presence of thermal conduction and cooling. We point out that these differences in the level of magnetic field are not due to the density variations between different cooling runs. With the exception of the very central regions, the differences in the density profiles are negligible (see Figure 9(a) below). Note also that the inclusion of cooling steepens the entropy gradient and flattens the temperature gradient over a range of radii, and both of these effects act to enhance the differences in the restoring forces in the runs with and without cooling. Thus, the differences in the field amplification are apparent when one compares the cooling-only run with the runs that include both cooling and conduction. Non-radiative counterparts of these runs do not show clear differences in the field amplification (see Figure 9(a) below).

In Figure 9 (left panel), we show the profiles of the magnetic field $B$ for the same bin size as in Figure 2. The color and symbol coding of all thick curves are the same as in Figure 2, and the thin curves correspond to the thick curve counterparts computed under the assumption that the amplification of the 
magnetic field $B_{\text {comp }}$ comes entirely from gas compression (i.e., $B_{\text {comp }} \propto \rho^{2 / 3}$ ), and the magnetic field is normalized such that the relative values of all curves in the figure are physically meaningful. While the magnetic field is in Gauss, we emphasize again that, technically speaking, the field values can be arbitrarily rescaled by varying the initial magnetic field as long as the field is weak (which is the case in our simulations). Within $r_{\text {vir }}\left(\sim 1.25 h^{-1} \mathrm{Mpc}\right)$ in the radiative cases, $B>B_{\text {comp }}$ and the average slope of the magnetic field $B$ very approximately

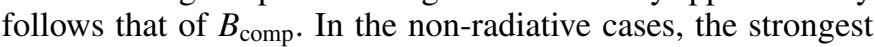
relative boost in the magnetic field amplification beyond pure compression occurs far from the cluster center. These trends are very similar to the ones observed by Dubois \& Teyssier (2008). The key difference is in the normalization of the actual magnetic field $B$, which is higher with respect to $B_{\text {comp }}$ in our simulations than that in Dubois \& Teyssier (2008). Interestingly, Dubois \& Teyssier (2008) find that the magnetic field in the center of the cluster in the non-radiative case is weaker than its compressional counterpart, and, quoting Roettiger et al. (1999), attribute this effect to numerical magnetic reconnection, noting that it is expected to be numerical scheme- and resolutiondependent. We also observe the flattening of the magnetic field profile in the center in the non-radiative case, and the decrease in the ratio of $B$ and $B_{\text {comp }}$ at small radii, but the field never becomes weaker than $B_{\text {comp }}$. As Dolag et al. (2005) find a strong dependence of the average magnetic field on the cluster mass and temperature, this difference can be entirely attributed to the fact that our cluster is hotter and more massive. Dolag et al. (2005) show that the excess of the magnetic field beyond that due to compression alone shows that induction/shearing plays an important role in the field amplification, and that in the most naive case of the amplification solely due to compression, no scaling of the average magnetic field with cluster mass would be present. Stronger shearing motions (and hence higher $B$ fields) in higher temperature clusters are likely the result of a longer merger history. The stronger excess of the magnetic field $B$ beyond $B_{\text {comp }}$ compared to that inferred by Dubois $\&$ Teyssier (2008) is entirely consistent with the fact that our cluster is over $\sim 2$ times more massive than that considered in their work. Using an approximate scaling $T_{\mathrm{vir}} \propto M_{\mathrm{vir}}^{2 / 3}$, this corresponds to an $\sim 1$. 7 change in virial temperature. Now, Dolag et al. (2002, 2005) demonstrate, albeit with different numerical technique, that such a change in cluster temperature (from $\sim 3.4 \mathrm{keV}$ to $5.75 \mathrm{keV}$ ) results in up to an order of magnitude higher average magnetic field independently of the resolution and whether the averaging is done within $0.1 r_{\text {vir }}$ or $r_{\text {vir }}$ (see Figure 2 in Dolag et al. 2002, 2005). Thus, the fact that we find a higher magnetic field amplification than in Dubois \& Teyssier (2008) is expected on physical grounds and is consistent with their work.

In Figure 9 (right panel), we show the magnetic field profiles for the case including anisotropic conduction and radiative cooling (red curves), and the non-radiative case with anisotropic conduction. Solid lines are for the seven levels of refinement, and dashed ones for the six levels of refinement. The bin size used in Figure 9 (left panel) was two times bigger than that in Figure 2. Figure 9 (left right) shows that, for the cases considered here, the adiabatic results are robust and do not show clear resolution trends. The magnitude of the field amplification in the adiabatic runs is comparable to that reported by Brüggen et al. (2005b). For cooling runs, the magnetic field increases with resolution. In general, an increase in the field strength with resolution is a recognized issue. Dolag et al. (2005) compared their older non-radiative simulations (Dolag et al. 2002) with new ones with 20 times higher mass resolution by presenting average magnetic fields in a sample of simulated clusters. They found that the average field within $0.1 r_{\text {vir }}$ is higher by up to $\sim 5$ times in clusters with temperatures less than $10 \mathrm{keV}$ in the better resolved runs. Similar results were obtained by Dolag \& Stasyszyn (2009) who varied mass resolution by a factor of 10 and found that the magnetic field profile values within $0.1 r_{\text {vir }}$ changed by up to $\sim 10$, and that the magnetic field levels increased with resolution. They also concluded that their profile shapes suggest more numerical dissipation compared to the FLASH code (Brüggen et al. 2005b) and slightly less than RAMSES (Dubois \& Teyssier 2008). However, we note that in the Brüggen et al. (2005b) simulation the magnetic field was by design dynamically decoupled from the gas dynamics. Dolag et al. (2002) show that averaging within $r_{\text {vir }}$ rather than $0.1 r_{\text {vir }}$ leads to significantly lower fields. As a side note we point out that, for radiative cooling cases, the magnetic field averaged within $r_{\mathrm{vir}}$ in our simulations would show diminished sensitivity to resolution. We point out that these comparisons are not perfect because the Dolag et al. $(2002,2005)$ simulations were performed with an entirely different numerical technique - smooth particle magneto-hydrodynamics - rather than using an AMR grid code. In any case, our radiative runs for seven levels of refinement may represent lower limits to the magnetic field strength. Finally, while the dependence of the magnetic field on resolution may be stronger in the runs including radiative cooling, the highest resolution runs at seven levels of refinement already show trends consistent with those presented by Dolag et al. (2005) and Dubois \& Teyssier (2008) as explained above. Performing runs at a resolution of eight levels of refinement and higher was not feasible using the techniques considered here.

\section{CONCLUSIONS}

We performed first magnetohydrodynamical simulations of cosmological galaxy cluster formation that simultaneously include magnetic fields, radiative cooling, and anisotropic thermal conduction. The presence of anisotropic conduction changes the properties of the ICM by making it convectively unstable independently of the sign of the ICM temperature gradient. In our approach, we self-consistently included the amplification of the magnetic field due to the shearing motions, gas compression enhanced by radiative cooling, and the kinematic dynamo associated with the anisotropic nature of conduction. Our key findings are as follows.

1. At large distances, turbulent motions tend to reset the (radial) orientation of the magnetic field that the MTI tries to establish. Nevertheless, some radial bias in the orientation of the field is seen at large radii in the runs with and without conduction. No clear excess of directional bias in the magnetic and velocity field is seen in the runs with anisotropic thermal conduction. The residual bias may be due to the infall of substructures, gas accretion along the filaments, and the inhomogeneous radial flows in the bulk of the ICM. However, radial bias is also expected when the MTI operates. The degree of this bias depends on the properties of the turbulence driven by structure formation, and disentangling the inhomogeneous radial gas flows and MTI scenarios is challenging. A systematic theoretical study of a number of clusters is required to quantify the role of MTI in shaping the magnetic field topology as the thermal and dynamical state of the ICM in other clusters (level of turbulent support, MTI growth rates, effective outer 

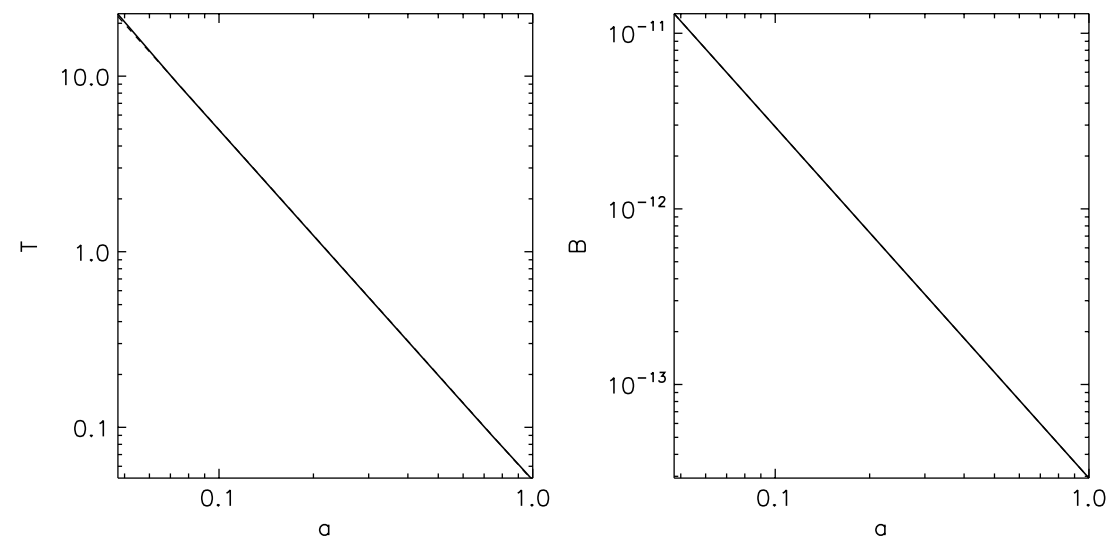

Figure 10. Evolution of physical temperature (left panel) and physical magnetic field with the cosmological expansion parameter $a$. Quantities on vertical axes in both panels are in arbitrary units. The test assumes uniform magnetic field, density, and temperature and neglects any seed fluctuations in the velocity field. The results are in perfect agreement with the theoretical scaling relations (power-law fits to the computed relations are also plotted and match these relations perfectly).

scale of turbulence driven by structure formation) will vary across the mass spectrum of clusters. Here, we report on the first study of the cosmological formation of one cluster that will form the basis for further investigation to address the above point.

2. Magnetic field amplification is significantly boosted in the presence of radiative cooling, which allows the gas to concentrate toward the cluster center. The central magnetic field at $z=0$ is amplified by over six orders of magnitude over the value obtained without substructure formation.

3. In the presence of thermal conduction and radiative cooling, the magnetic field is amplified by a kinematic dynamo process beyond the values obtained in the cooling-only run. This additional amplification occurs over a broad range of radii. The restoring forces in the conducting fluid are proportional to the temperature gradient rather than the (steeper) entropy gradient, which makes it easier to mix the ICM and amplify the fields by the continuous substructure infall.

4. The effective heat conduction from the hotter outer layers of the cluster to its center is reduced below the full Braginskii-Spitzer value. However, the effective radiative cooling driven accretion is noticeably reduced.

5. The radiative run with anisotropic thermal conduction exhibits a tendency for a tangential bias in the velocity and magnetic fields within the inner $\sim 0.3 h^{-1} \mathrm{Mpc}$ that could be associated with HBI or trapped gravity modes. Future higher resolution cosmological cluster formation studies will assess the robustness of this effect. Any possible anisotropy in the magnetic field distribution may be detectable via radio polarization measurements with the Square Kilometer Array and LOFAR, while the bias in the velocity field could be probed with the future International $X$-ray Observatory mission.

We thank the referee for a very constructive and detailed report that helped us to significantly improve the paper. The software used in this work was in part developed by the DOEsupported ASC/Alliance Center for Astrophysical Thermonuclear Flashes at the University of Chicago. M.R. thanks the staff of the NASA Ames Research center for technical help with performing the runs at the Pleiades supercomputer where most of the runs were performed. We are indebted to Chris Daley for his assistance with the particle and gravity modules in the FLASH

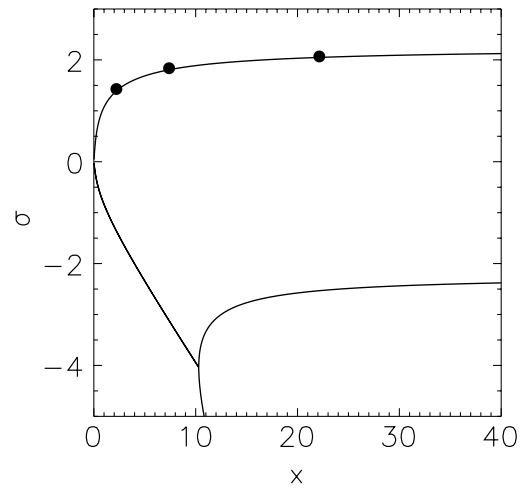

Figure 11. Magnetothermal instability growth rate in units of Brunt-Väisälä frequency as a function of $x=(\gamma-1) \kappa(T / P) k^{2} / \omega_{\mathrm{BV}}$, where $k$ is the wavenumber of the velocity fluctuation and $\omega_{\mathrm{BV}}$ is the Brunt-Väisälä frequency. Solid line denotes the prediction from linear theory and the dots show the results obtained with the FLASH code.

code. We thank Eliot Quataert, Maxim Markevitch, Christoph Pfrommer, Paul Nulsen, Christine Jones, Larry David, Bill Forman, Milos Milosavljevic, John ZuHone, Mikhail Medvedev, Steve Balbus, and Fabian Heitsch for discussions.

\section{APPENDIX A}

In order to check the implementation of the cosmological terms in the MHD equations, we evolved spatially constant matter density and magnetic field while neglecting any velocity perturbations. The result of this test is shown in Figure 10. In the left panel we show the scaling of the temperature (in arbitrary units) with the cosmological expansion factor $a$. Shown are the code result for the physical temperature and the power-law fit (solid and dashed lines are practically indistinguishable). The slope of $T(a) \propto a^{-2}$ agrees with the standard theoretical expectation. The right panel shows the physical magnetic field in arbitrary units as a function of the scaling parameter $a$ and a power-law fit to this relation, both as a function of the scaling parameter $a$. Here again the fit is perfect, the solid and dashed lines overlap, and the slope of the field is $\left(B(a) \propto a^{-2}\right)$, ensuring the conservation of the magnetic flux.

\section{APPENDIX B}

In order to test the implementation of the anisotropic thermal conduction module we compared linear theory MTI growth rates 

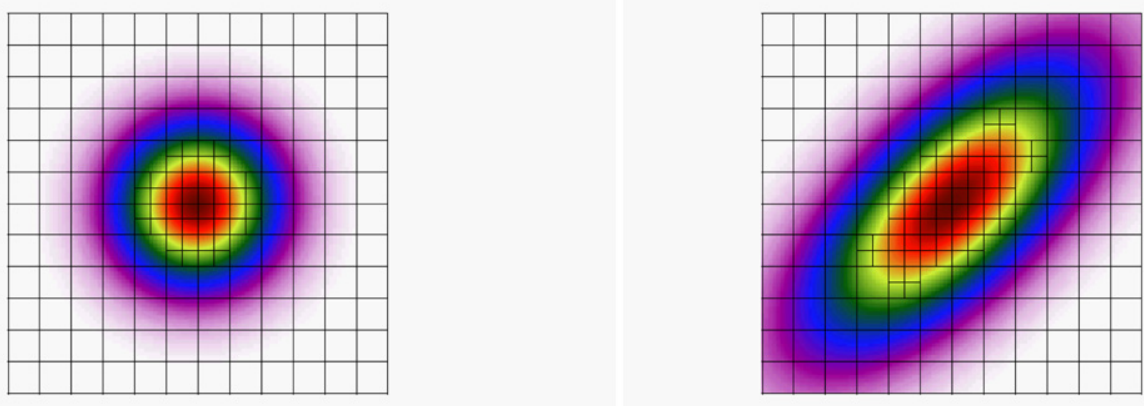

Figure 12. Test of anisotropic diffusion of a passive scalar along diagonal magnetic fields. Adaptive mesh is superimposed on the fluid distribution. Each grid element is divided into a number of individual zones. Initial and final states are shown in the left and right panels, respectively.

(A color version of this figure is available in the online journal.)

with the code results. This test is very similar to the one discussed in Parrish \& Stone (2005). That is, we set up a two-dimensional stratified hydrostatic atmosphere with very shallow density and temperature profiles such that

$$
\begin{gathered}
T(z)=T_{o}\left(1-y / y_{o}\right) \\
\rho(z)=\rho_{o}\left(1-y / y_{o}\right)^{2},
\end{gathered}
$$

where $T_{o}$ and $\rho_{o}$ are constants. The characteristic lengthscale $y_{o}$ was set to $1 \%$ of the horizontal height of the computational box. The gravitational field was assumed constant throughout the computational domain. We set hydrostatic boundary conditions on the top and bottom boundaries. We also impose constant temperature in the boundary zones to prevent the escape of thermal energy from the simulation box via thermal conduction. That is, the computational domain is effectively adiabatic. We set periodic boundary conditions in the horizontal direction. The initial magnetic field is horizontal with very high magnetic $\beta$ parameter. In order to seed the instability, we introduce a very small sinusoidal velocity perturbation in the vertical direction. Specifically, the initial vertical component of the velocity field is given by

$$
v_{z}(y)=v_{o} \sin (2 \pi y / L)
$$

where $v_{o}$ is a very small constant amplitude of the velocity perturbation and $L$ is the box size in the horizontal $y$-direction. We evolve such initial conditions and, using the velocity field as a function of time, we compute the instability growth rate $\sigma$ following a prescription similar to that in Parrish \& Stone (2005). These results are then compared to the linear theory prediction in the nondimensional form

$$
\sigma^{3}+\frac{1}{\gamma} \sigma^{2} x+\sigma+\frac{d \ln T}{d \ln S} x=0
$$

where $\sigma$ is the instability growth rate in units of the Brunt-Väisälä frequency $\omega_{\mathrm{BV}}$ and

$$
x=(\gamma-1) \kappa \frac{T}{P} k^{2} \omega_{\mathrm{BV}}^{-1},
$$

where $k$ is the wavenumber of the velocity fluctuation. The result of this test is shown in Figure 11. The growth rates predicted by the code are in excellent agreement with the theoretical expectations. In Figure 12, we show the results of a test of the anisotropic diffusion of a passive scalar. In this test, the passive fluid evolves entirely due to the diffusion along the magnetic field lines. That is, this test isolates the effects introduced by the anisotropic diffusion module to demonstrate its robustness in the presence of the AMR. The left panel shows the initial state, and the right one the final evolved state. The adaptive grid is superimposed on the fluid distribution. Note that each grid element is further divided into a number of individual zones. Magnetic fields are uniform and diagonal. This test clearly demonstrates that the adaptive mesh follows the distribution of the fluid and that no artifacts are introduced by the anisotropic diffusion module at the mesh refinement interfaces.

\section{REFERENCES}

Balbus, S. A. 2000, ApJ, 534, 420

Bogdanović, T., Reynolds, C. S., Balbus, S. A., \& Parrish, I. J. 2009, ApJ, 704, 211

Böhringer, H., \& Werner, N. 2010, A\&ARv, 18, 127

Brüggen, M., Ruszkowski, M., \& Hallman, E. 2005a, ApJ, 630, 740

Brüggen, M., Ruszkowski, M., Simionescu, A., Hoeft, M., \& Dalla Vecchia, C. 2005b, ApJ, 631, L21

Cavagnolo, K. W., Donahue, M., Voit, G. M., \& Sun, M. 2009, ApJS, 182, 12

Chandran, B. D., \& Dennis, T. J. 2006, ApJ, 642, 140

Churazov, E., Forman, W., Jones, C., \& Böhringer, H. 2003, ApJ, 590, 225

Collins, D., Xu, H., Norman, M., Li, H., \& Li, S. 2009, ApJS, 186, 308

Cowie, L. L., \& McKee, C. F. 1977, ApJ, 211, 135

Dolag, K., Bartelmann, M., \& Lesch, H. 1999, A\&A, 348, 351

Dolag, K., Bartelmann, M., \& Lesch, H. 2002, A\&A, 387, 383

Dolag, K., Grasso, D., Springel, V., \& Tkachev, I. 2005, J. Cosmol. Astropart. Phys., JCAP01(2005)009

Dolag, K., Jubelgas, M., Springel, V., Borgani, S., \& Rasia, E. 2004, ApJ, 606, L97

Dolag, K., \& Stasyszyn, F. 2009, MNRAS, 398, 1678

Dougal, A. A., \& Goldstein, L. 1958, Phys. Rev., 109, 615

Dubois, Y., Devriendt, J., Slyz, A., \& Silk, J. 2009, MNRAS, 399, L49

Dubois, Y., \& Teyssier, R. 2008, A\&A, 482, L13

Dursi, L. J., \& Pfrommer, C. 2008, ApJ, 677, 993

Enßlin, T. A., \& Vogt, C. 2003, A\&A, 401, 835

Evrard, A. E. 1990, ApJ, 363, 349

Fabian, A. C., Sanders, J. S., Allen, S. W., et al. 2003, MNRAS, 344, L43

Feretti, L., \& Johnston-Hollitt, M. 2004, New Astron. Rev., 48, 1145

Finoguenov, A., Ruszkowski, M., Jones, C., et al. 2008, ApJ, 686, 911

Forman, W., Nulsen, P., Heinz, S., et al. 2005, ApJ, 635, 894

Frenk, C. S., White, S. D. M., Bode, P., et al. 1999, ApJ, 525, 554

Guo, F., Oh, S. P., \& Ruszkowski, M. 2008, ApJ, 688, 859

Heinz, S., Brüggen, M., \& Friedman, S. 2011, ApJS, 194, 21

Jubelgas, M., Springel, V., \& Dolag, K. 2004, MNRAS, 351, 423 
Kunz, M. W., Schekochihin, A. A., Cowley, S. C., Binney, J. J., \& Sanders, J. S 2011, MNRAS, 410, 2446

Lee, D., \& Deane, A. E. 2009, J. Comput. Phys., 228, 952

Lee, D., Deane, A. E., \& Federrath, C. 2009, in ASP Conf. Ser. 406, Numerical Modeling of Space Plasma Flows: ASTRONUM-2008, ed. N. V. Pogorelov et al. (San Francisco, CA: ASP), 243

Li, S., Li, H., \& Cen, R. 2008, ApJS, 174, 1

Lufkin, E. A., Balbus, S. A., \& Hawley, J. F. 1995, ApJ, 446, 529

Lufkin, E. A., Sarazin, C. L., \& White, III, R. E. 2000, ApJ, 542, 94

Mahdavi, A., Hoekstra, H., Babul, A., Balam, D. D., \& Capak, P. L. 2007, ApJ, 668,806

Markevitch, M., Gonzalez, A. H., David, L., et al. 2002, ApJ, 567, L27

Markevitch, M., \& Vikhlinin, A. 2007, Phys. Rep., 443, 1

McCourt, M., Parrish, I. J., Sharma, P., \& Quataert, E. 2011, MNRAS, 413 1295

McNamara, B. R., \& Nulsen, P. E. J. 2007, ARA\&A, 45, 117

Medvedev, M. V. 2007, ApJ, 662, L11

Nagai, D., Kravtsov, A. V., \& Kosowsky, A. 2003, ApJ, 587, 524

Narayan, R., \& Medvedev, M. V. 2001, ApJ, 562, L129

Norman, M. L. 2010, arXiv:1005.1100

O’Neill, S. M., DeYoung, D. S., \& Jones, T. W. 2009, ApJ, 694, 1317

Padmanabhan, P. 2000, Theoretical Astrophysics, Vol. 1: Astrophysical Processes (Cambridge: Cambridge Univ. Press), 601

Parrish, I. J., \& Quataert, E. 2008, ApJ, 677, L9

Parrish, I. J., Quataert, E., \& Sharma, P. 2010, ApJ, 712, L194

Parrish, I. J., \& Stone, J. M. 2005, ApJ, 633, 334

Parrish, I. J., Stone, J. M., \& Lemaster, N. 2008, ApJ, 688, 905

Pfrommer, C., \& Dursi, J. L. 2010, Nat. Phys., 6, 520

Quataert, E. 2008, ApJ, 673, 758

Rasera, Y., \& Chandran, B. 2008, ApJ, 685, 105

Reynolds, C. S., McKernan, B., Fabian, A. C., Stone, J. M., \& Vernaleo, J. C. 2005, MNRAS, 357, 242
Ricker, P. M., Dodelson, S., \& Lamb, D. Q. 2000, ApJ, 536, 122

Robinson, K., Dursi, L. J., Ricker, P. M., et al. 2004, ApJ, 601, 621

Roettiger, K., Burns, J. O., \& Stone, J. M. 1999, ApJ, 518, 603

Ruszkowski, M., \& Begelman, M. C. 2002, ApJ, 581, 223

Ruszkowski, M., Brüggen, M., \& Begelman, M. C. 2004a, ApJ, 611, 158

Ruszkowski, M., Brüggen, M., \& Begelman, M. C. 2004b, ApJ, 615, 675

Ruszkowski, M., Enßlin, T. A., Brüggen, M., Begelman, M. C., \& Churazov, E. 2008, MNRAS, 383, 1359

Ruszkowski, M., Enßlin, T. A., Brüggen, M., Heinz, S., \& Pfrommer, C. 2007, MNRAS, 378, 662

Ruszkowski, M., \& Oh, S. P. 2010, ApJ, 713, 1332

Ruszkowski, M., \& Oh, S. P. 2011, MNRAS, 414, 1493

Sanders, J. S., Fabian, A. C., Smith, R. K., \& Peterson, J. R. 2010, MNRAS, 402, L11

Sanderson, A. J. R., O’Sullivan, E., \& Ponman, T. J. 2009, MNRAS, 395, 764

Schekochihin, A. A., \& Cowley, S. C. 2007, Turbulence and Magnetic Fields in Astrophysical Plasmas (Berlin: Springer), 85

Schekochihin, A. A., Cowley, S. C., Rincon, F., \& Rosin, M. S. 2010, MNRAS, 405, 291

Sharma, P., Chandran, B. D. G., Quataert, E., \& Parrish, I. J. 2009, ApJ, 699, 348

Sharma, P., \& Hammett, G. W. 2007, J. Comput. Phys., 227, 123

Sijacki, D., \& Springel, V. 2006, MNRAS, 371, 1025

Sutherland, R. S., \& Dopita, M. A. 1993, ApJS, 88, 253

Tozzi, P., \& Norman, C. 2001, ApJ, 546, 63

Vazza, F., Brunetti, G., Kritsuk, A., et al. 2009, A\&A, 504, 33

Vogt, C., \& Enßlin, T. A. 2003, A\&A, 412, 373

Voigt, L. M., \& Fabian, A. C. 2004, MNRAS, 347, 1130

Voit, G. M., Cavagnolo, K. W., Donahue, M., et al. 2008, ApJ, 681, L5

Werner, N., et al. 2010, MNRAS, 407, 2063

Xu, H., Li, H., Collins, D. C., Li, S., \& Norman, M. L. 2009, ApJ, 698, L14

ZuHone, J. A., Markevitch, M., \& Johnson, R. E. 2010, ApJ, 717, 908 